\title{
Robust Aluminium and Iron Phosphinate Metal Organic Frameworks for Efficient Removal of Bisphenol A
}

\author{
Daniel Bůžek, ${ }^{\mathrm{a}, \mathrm{b}}$ Soňa Ondrušová, ${ }^{\mathrm{a}}$ Jan Hynek, ${ }^{\mathrm{a}}$ Petr Kovář, ${ }^{\mathrm{c}}$ Kamil Lang, ${ }^{\mathrm{a}}$ Jan Rohlíček, ${ }^{\mathrm{d}}$ Jan De- \\ $\mathrm{mel}^{\mathrm{a}^{*}}$ \\ ${ }^{a}$ Institute of Inorganic Chemistry of the Czech Academy of Sciences, 25068 Husinec-Řež, Czech Republic; E-mail: \\ demel@iic.cas.cz \\ ${ }^{\mathrm{b}}$ Faculty of Environment, Jan Evangelista Purkyně University, Králova Výšina 3132/7, 40096 Ústí nad Labem, Czech \\ Republic \\ ${ }^{\mathrm{c} C h a r l e s ~ U n i v e r s i t y, ~ F a c u l t y ~ o f ~ M a t h e m a t i c s ~ a n d ~ P h y s i c s, ~ K e ~ K a r l o v u ~ 3, ~ 121 ~ 16, ~ P r a h a ~ 2, ~ C z e c h ~ R e p u b l i c ~}$ \\ ${ }^{\mathrm{d}}$ Institute of Physics of the Czech Academy of Sciences, 18221 Praha, Czech Republic
}

\begin{abstract}
Porous metal-organic frameworks (MOFs) have excellent characteristics for the adsorptive removal of environmental pollutants. Herein, we introduce a new series of highly stable MOFs, constructed using $\mathrm{Fe}^{3+}$ and $\mathrm{Al}^{3+}$ metal ions and bisphosphinate linkers. The isoreticular design leads to ICR-2, ICR-4, ICR-6, and ICR-7 MOFs with a honeycomb arrangement of linear pores, surface areas up to $1360 \mathrm{~m}^{2} \mathrm{~g}^{-1}$, and high solvothermal stability. In most cases, their sorption capacity is retained even after $24 \mathrm{~h}$ reflux in water. The choice of the linkers allows fine tuning of the pore sizes and the chemical nature of the pores. This feature can be utilized for optimization of host-guest interactions between molecules and pore walls. Water pollution by various endocrine disrupting chemicals has been considered as a global threat to public health. In this work, we proved that the chemical stability and the hydrophobic nature of the synthesized series of MOFs result in remarkable sorption properties of these materials for neurodisruptor bisphenol A.
\end{abstract}

\section{INTRODUCTION}

Endocrine disrupting chemicals are gaining increased attention among emerging pollutants due to their influence on endocrine system by mimicking or blocking natural hormones, or over/under production of specific hormones. ${ }^{1}$ This diverse group of pollutants is utilized in broad spectrum of human products such as herbicides (DDT, Propanil), antimicrobial agents (Triclosan), detergents, toiletries, cosmetics (parabens, phenols), pharmaceutics (diethystilbestrol), and plastics (phthalates, bisphenol A, brominated flame retardants) ${ }^{1,2}$ The most studied endocrine disruptor is bisphenol A (2,2-bis(4hydroxyphenyl)propane; BPA). Over a million tons per year of BPA is produced mainly for the production of plastics such as polycarbonate and epoxy resins. It was found that BPA can leach from the products (e.g., beverage containers and packages, baby bottles, dental sealants), migrate into the environment, and enter the food chain. $^{2-4}$ Therefore, methods for fast and effective removal of BPA from waste water or landfill leachate are of great importance. Commonly used sorbents, such as activated charcoal, zeolites, or clays have low affinity toward BPA or low sorption capacity. ${ }^{5,6}$ For these reasons, new materials have to be developed in order to stop spreading endocrine disruptors in the environment.

Metal-organic frameworks (MOFs) belong to the fast growing area of organic-inorganic hybrids. MOFs are combining metal nodes (secondary building units - SBUs) and polytopic organic ligands (linkers). ${ }^{7}$ The number of possible SBUs and organic linkers gives rise to thousands of new structures with varying topologies, pore sizes, and chemical compositions. ${ }^{8-10}$ The high surface area of MOFs, ${ }^{11,12}$ along with the possibility of introducing functional groups, ${ }^{1,14}$ make them highly promising materials for many applications, ${ }^{15}$ including sorbents of pollutants. ${ }^{16-18}$ Unfortunately even after decades of extensive research, water stability of MOFs still remains an issue, ${ }^{19}$ thus limiting their applicability for waste water treatment.

Recently, we have demonstrated that the combination of $\mathrm{Fe}^{3+}$ ions with bisphosphinate linker $\mathrm{H}_{2} \mathrm{PBP}(\mathrm{Me}$ ) (Figure 1) leads to a new MOF, named Fe-ICR-2 (ICR stands for Inorganic Chemistry Řež), with a honeycomb structure. $^{20}$ Importantly, Fe-ICR-2 is endowed with higher hydrothermal stability in comparison with a carboxylatebased analogue Fe-MIL-53. ${ }^{21}$ The stability is related to the stronger coordination bond of phosphinates to hard metals, such as $\mathrm{Fe}^{3+}$, in comparison with carboxylate groups. Notably, the methyl group bound at phosphorus atom is pointing into the volume of the Fe-ICR-2 pore. Substitution with bulkier phenyl group leads to decrease in the pore size (Fe-ICR-4), ${ }^{20}$ suggesting that this strategy can allow for fine-tuning of pore hydrophobicity.

In this work, we applied the reticular design and extended the bisphosphinate linkers. We synthesized and 
delineated the properties of a series of phosphinate MOFs denoted ICR-2, ICR-4, ICR-6, and ICR-7 in both $\mathrm{Fe}^{3+}$ and $\mathrm{Al}^{3+}$ versions (Figure 1). All prepared MOFs possess the honeycomb arrangement of linear pores with sizes varying from $3 \AA$ to $24 \AA$. The hydrophobic nature of the pores leads to the high sorption capacity for BPA.

\section{RESULTS AND DISCUSSION}

The solvothermal reaction of bisphosphinate linkers (Figure 1, bottom-left) with $\mathrm{FeCl}_{3} \cdot 6 \mathrm{H}_{2} \mathrm{O}$ or $\mathrm{AlCl}_{3} \cdot 6 \mathrm{H}_{2} \mathrm{O}$ in EtOH at $250{ }^{\circ} \mathrm{C}$ yielded crystalline ICR MOFs. The only exception was the reaction of $\mathrm{H}_{2} \mathrm{BBP}(\mathrm{Me})$ with $\mathrm{FeCl}_{3} \cdot 6$ $\mathrm{H}_{2} \mathrm{O}$ which led to a nonporous layered material Fe-ICR-5. In this case, the synthetic conditions were optimized and porous Fe-ICR- 6 was obtained in DMF at $120{ }^{\circ} \mathrm{C}$ after three-day reaction. However, Fe-ICR-6 is of inferior crystallinity due to lower temperature used when compared with other ICR MOFs (see below). The composition of all synthesised MOFs is given in Table 1 and is confirmed by elemental analyses and FTIR spectra (Table S1, Figures S1$\left.\mathrm{S}_{7}\right)$.

The thermal stability of ICR MOFs in air was investigated by thermogravimetric analyses in conjunction with differential thermal analyses and mass spectroscopy (TGA/DTA/MS) (Figures S8-S14). The TGA curves indicate that all prepared ICR MOFs are endowed with high thermal stability and contain negligible amount of solvent or water molecules inside the pores. The least stable is $\mathrm{Al}$ ICR-4 which starts to decompose at $35^{\circ}{ }^{\circ} \mathrm{C}$, whereas $\mathrm{Al}$ ICR-7 is the most thermally stable ICR MOF with decomposition temperature of $550^{\circ} \mathrm{C}$.

The crystal structure of Fe-ICR-7 was obtained from powder X-ray diffraction (PXRD) data (Table S2). The indexing was performed in the DICVOLo6 program $^{22}$ and the crystal structure models were found $a b$ initio using the FOX software (detailed description is in the Supporting Information. ${ }^{23}$ The final Rietveld fit confirms the proposed structure (Table S2, Figure S16). The low crystallinity of Fe-ICR-6 did not allow indexing of the PXRD pattern. Nevertheless, the isoreticular design of ICR MOFs and solved structures of Fe-ICR-2 and Fe-ICR- $4{ }^{20}$ enabled creation of a structural model followed by geometry optimization using the PCFF force field and the Rietveld refinement in the Materials Studio software (Figure S18). ${ }^{24}$ The PXRD patterns of all Fe-ICR MOFs are compared in Figure 1, bottom-middle.

Motivated by successful syntheses of Fe-ICR MOFs, we investigated the structural arrangements of aluminiumbased ICR MOFs (Al-ICR MOFs). In the case of Al-ICR-4, the quality of the PXRD pattern allowed solving the crystal structure $a b$ initio using the Superflip package ${ }^{25}$ with the histogram matching option (Figure $\mathrm{S}_{17}$ ). The detailed analysis of the corresponding PXRD pattern confirms nearly identical crystal structure of Al-ICR-4 with that of Fe-ICR-4 ${ }^{20}$ (Table S2). In general, the analyses of PXRD patterns of Al-ICR MOFs revealed that they form identical structural motifs for each linker with those of Fe-ICR MOFs (Figure S2o).

As illustrated for Fe-ICR MOFs (Figure 1 , middle),$^{20}$ the secondary building units (SBUs) of Fe-ICR MOFs and AlICR MOFs (not applicable for Fe-ICR-5, see below for details) are composed of octahedrally coordinated metal atoms bound together through O-P-O bridges forming one-dimensional $(\mathrm{lD})$ infinity columns. The columns are connected via phenylene or biphenylene bridges forming the three-dimensional $(3 \mathrm{D})$ honeycomb framework. Feand $\mathrm{Al}-$ versions of ICR- 6 and ICR-7 are isoreticular structures to Fe-ICR-2 with increased pore sizes due to the incorporated biphenylene spacer. The crystal structures of Fe-ICR-4 and Al-ICR-4 are constructed similarly to the structure of Fe-ICR-2, i.e., the honeycomb arrangement is composed of $1 \mathrm{D}$ infinity columns tied together by O-P-O bridges. However, the phenylene groups connecting the ${ }_{1} \mathrm{D}$ columns are not parallel to each other, but they are crossed and rotated in neighbouring layers (Figure 1 left). 


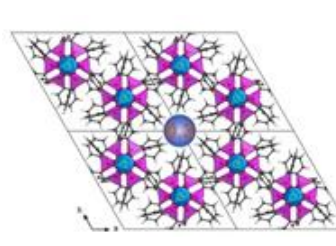

ICR-4
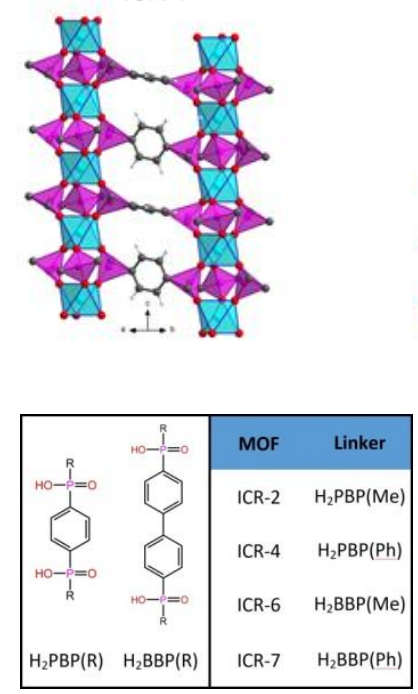

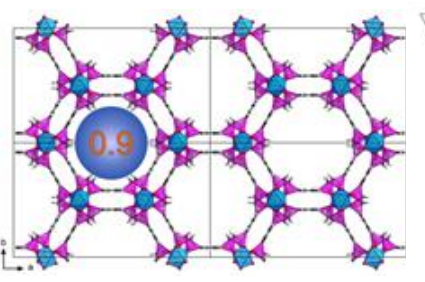

ICR-2
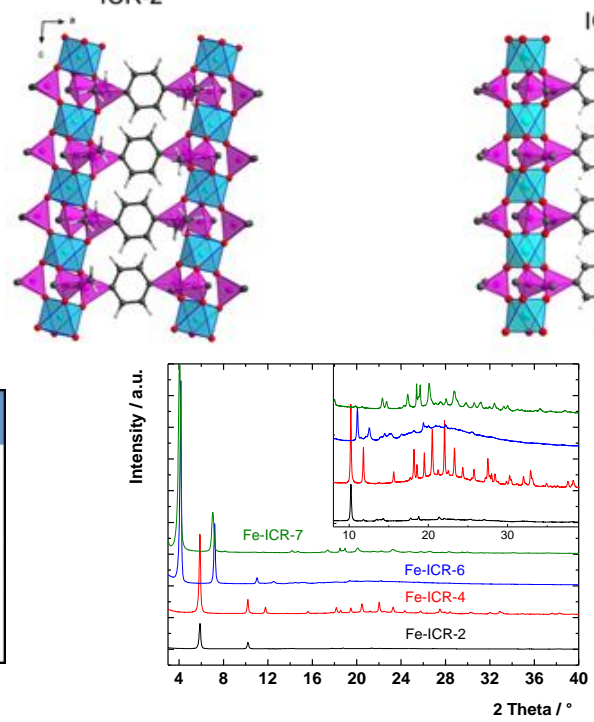
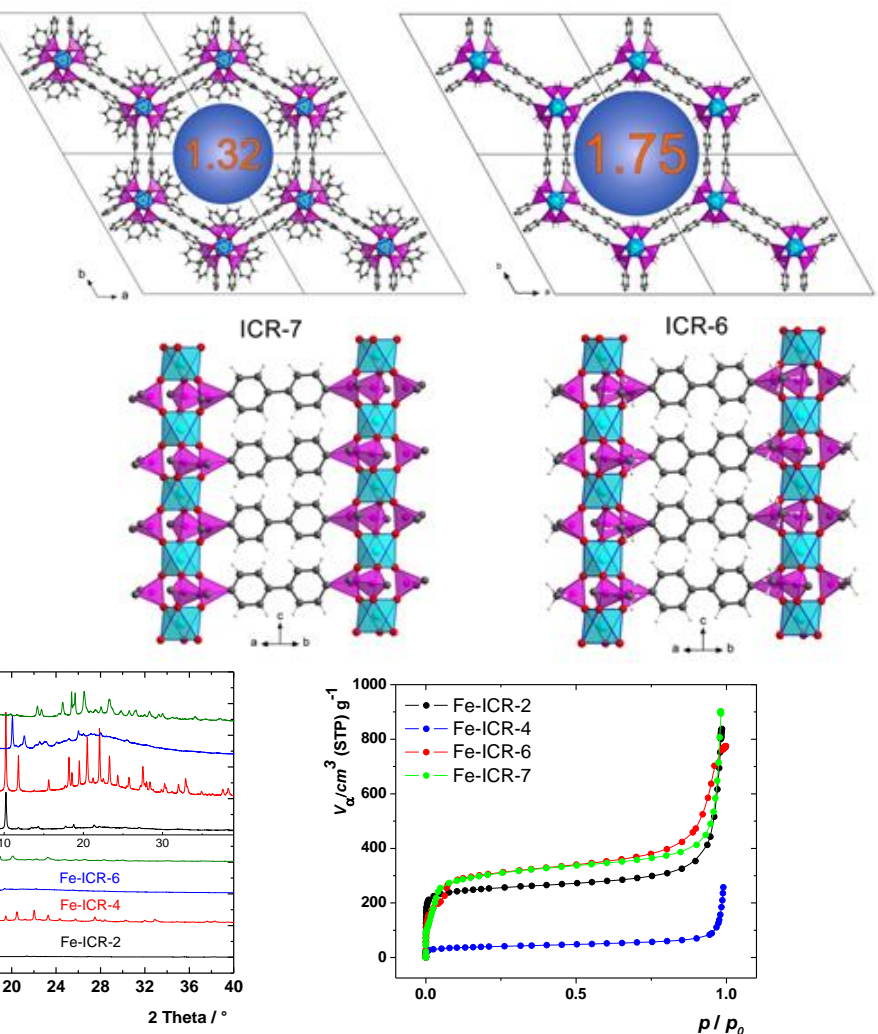

Figure 1. Honeycomb patterns of $1 \mathrm{D}$ pores of Fe-ICR MOFs running along the $c$-axis (top), the pore limiting diameter (nm) calculated by Poreblazer is indicated in the middle of the pore; $1 \mathrm{D}$ columns of octahedrally coordinated iron atoms bridged by phosphinate acid groups (middle), ICR MOFs coding (bottom-left), PXRD of Fe-ICR MOFs (bottom-middle), and adsorption isotherms of nitrogen for Fe-ICR MOFs (bottom-right). Colour coding: octahedrally coordinated iron atoms (blue), phosphinate tetrahedra (magenta), O (red), C (grey), and $\mathrm{H}$ (white).

The crystal structure of Fe-ICR-5 was also solved from PXRD data in this work (Table S2, Figure S15). Its structure is layered, composed of $1 \mathrm{D}$ infinity columns of iron atoms coordinated by O-P-O bridges (Figure S19). In this case, oxygen atoms are coordinated to $\mathrm{Fe}^{3+}$ centres in a trigonal bipyramid formation and every two neighbouring bipyramids are edge-sharing. In the chain, the pairs of edge-shared bipyramids are connected through vertices by four phosphinate tetrahedrons. The chains form bilayers that are held together only by weak nonbonding interactions. This structural arrangement is isoreticular to Fe-ICR- $3 .{ }^{20}$ Comparison of both structures is given in Figure S19. Since Fe-ICR-5 is nonporous this material was not further investigated.

The permanent porosity of activated ICR MOFs was probed by measurement of $\mathrm{N}_{2}$ adsorption isotherms at 77 K (Figure 1, bottom-right and Figure $\mathrm{S}_{43}$ ). All adsorption isotherms display a steep $\mathrm{N}_{2}$ uptake at low $\mathrm{P} / \mathrm{P}_{\mathrm{o}}$ ratios which is typical for microporous materials. More specifically, ICR-4 contains ultramicropores, whereas the pore diameters of ICR- 6 and ICR-7 are at the borderline between micropores and mesopores (Table 1 and Figures S21-24).
To better understand the porous structure of ICR MOFs, these MOFs were computationally analysed using the Poreblazer software ${ }^{26,27}$ for $\mathrm{N}_{2}$ molecule with $3.314 \AA$ in diameter (Table 1). The obtained parameters for Fe-ICR-2 and Al-ICR-2 are in agreement with the experimental values. The calculated pore limiting diameters (PLD) of Fe-ICR-4 and Al-ICR-4 are of $2.9 \AA$ 年; therefore, the accessible surface area cannot be calculated. Nevertheless, the pores are still accessible to $\mathrm{N}_{2}$ as evidenced by the corresponding adsorption isotherms (Figure 1, bottom-right and Figure $\mathrm{S}_{43}$ ). The BET specific surface areas of Fe-ICR7 and Al-ICR-7 fit well the calculated values, whereas in the case of Fe-ICR- 6 and Al-ICR-6 the BET specific surface areas are considerably lower, probably due to lower crystallinity and/or pore blocking. The PLDs of Fe-ICR-6 and Al-ICR-6 and Fe-ICR-7 and Al-ICR-7 are considerably smaller than pore diameters obtained by the NLDFT method from adsorption isotherms. This difference can be caused by roughness of pore walls decreasing the smallest opening of the pores, and/or by the hydrophobic nature of the pores significantly differing from the chemical nature used by the kernel in the NLDFT method. 
Table 1. Specific surface area, pore diameter, calculated pore limiting diameter, and accessible surface area for all synthetized MOFs

\begin{tabular}{|c|c|c|c|c|c|c|c|}
\hline Sample & Linker & $\begin{array}{c}\text { Specific } \\
\text { Surface area } \\
\text { Area } \\
{\left[\mathrm{m}^{2} \mathrm{g-}^{-1}\right]}\end{array}$ & $\begin{array}{l}\text { Pore Diameter } \\
{[\mathrm{nm}]^{\mathrm{a}}}\end{array}$ & $\begin{array}{l}\text { Pore Volume } \\
{\left[\mathrm{cm}^{3} \mathrm{~g}^{-1}\right]^{\mathrm{b}}}\end{array}$ & $\begin{array}{c}\text { Pore Limiting } \\
\text { Diameter } \\
{[\mathrm{nm}]^{\mathrm{c}}}\end{array}$ & $\begin{array}{c}\text { Accessible } \\
\text { Surface Area } \\
{\left[\mathrm{m}^{2} \mathrm{~g}^{-1}\right]^{\mathrm{d}}}\end{array}$ & $\begin{array}{l}\text { Pore Accessible } \\
\text { Volume }\left[\mathrm{cm}^{3} \mathrm{~g}^{-1}\right]^{\mathrm{e}}\end{array}$ \\
\hline Fe-ICR-2 & $\mathrm{H}_{2} \mathrm{PBP}(\mathrm{Me})$ & $906^{f}$ & 0.71 & 0.39 & 0.90 & 850 & 0.48 \\
\hline Fe-ICR-4 & $\mathrm{H}_{2} \mathrm{PBP}(\mathrm{Ph})$ & $165^{f}$ & n.a. & 0.044 & 0.29 & o & n.a. \\
\hline Fe-ICR-6 & $\mathrm{H}_{2} \mathrm{BBP}(\mathrm{Me})$ & $1134^{g}$ & 2.39 & 1.32 & 1.75 & 1562 & 1.00 \\
\hline Fe-ICR-7 & $\mathrm{H}_{2} \mathrm{BBP}(\mathrm{Ph})$ & $1125^{\mathrm{g}}$ & 2.16 & 0.79 & 1.32 & 1097 & 0.64 \\
\hline Al-ICR-2 & $\mathrm{H}_{2} \mathrm{PBP}(\mathrm{Me})$ & $933^{f}$ & 0.74 & 0.44 & 0.90 & 876 & 0.48 \\
\hline Al-ICR-4 & $\mathrm{H}_{2} \mathrm{PBP}(\mathrm{Ph})$ & $190^{f}$ & n.a. & 0.055 & 0.29 & o & n.a. \\
\hline Al-ICR-6 & $\mathrm{H}_{2} \mathrm{BBP}(\mathrm{Me})$ & $1362^{g}$ & 2.34 & 1.59 & 1.75 & 1660 & 1.06 \\
\hline Al-ICR-7 & $\mathrm{H}_{2} \mathrm{BBP}(\mathrm{Ph})$ & $1030^{g}$ & 2.07 & 1.52 & 1.31 & 1088 & 0.63 \\
\hline
\end{tabular}

${ }^{a}$ Pore diameter obtained by the MP plot for ICR-2 and ICR-4, otherwise by the NLDFT method; ${ }^{\mathrm{b}}$ Total pore volume; ${ }^{\mathrm{c}}$ Pore limiting diameter calculated by the Poreblazer software; ${ }^{\mathrm{d}}$ Accessible surface area calculated by the Poreblazer software; ${ }^{\mathrm{e}}$ Accessible pore volume calculated by the Poreblazer software; ${ }^{\mathrm{f}}$ Specific surface area calculated by the t-plot; ${ }^{\mathrm{g}}$ BET specific surface area.

Synthesized ICR MOFs are expected to be chemically stable under harsh conditions as described previously for Fe-ICR-2. ${ }^{20}$ After treatment of ICR MOFs in water, EtOH, and toluene at RT or under reflux, the PXRD patterns of most of ICR MOFs remained unchanged, suggesting preservation of the crystallinity and original structure (Figures S25-S42). Only Fe-ICR-6, Al-ICR-6, and Al-ICR-7 recrystallized or lost crystallinity in boiling water. Generally, the longer the linker, the lower is the stability of MOFs. For example, UiO-66 built of the terephthalate linker is stable in water and under humid atmosphere, however, both UiO-67 and UiO-68 made of biphenyl-4,4'dicarboxylate and p-terphenyl-4,4"-dicarboxylate linkers, respectively, decompose when exposed to humid air. ${ }^{28}$ This behaviour is not the case of presented ICR MOFs.

We also analysed the effects of these treatments on specific surface area (Table 2, Figures $\mathrm{S}_{44}$-S61), except for Fe-ICR-4 and Al-ICR-4. In these two cases, small molecules, such as water, block the pores and are not removed even during activation $\left(150^{\circ} \mathrm{C}\right.$, vacuum, $\left.24 \mathrm{~h}\right)$, while keeping the PXRD patterns intact. Fe-ICR-2, Al-ICR-2, Fe-ICR6, Fe-ICR-7, and Al-ICR-7 retained their porosity in the tested solvents at RT, in boiling toluene, and with the exception of Fe-ICR-2 in boiling EtOH. Interestingly, Al-
ICR-6 behaves differently. The specific surface area decreased after the solvent treatments at RT, whereas the treatment in boiling EtOH or toluene resulted in an increase of the specific surface area, probably due to the formation of structural defects. In general, boiling water represents one of the most challenging condition for MOFs. In this respect, Fe-ICR-2, Al-ICR-2, and Fe-ICR-7 preserve the majority of their porosity. Clearly, both $\mathrm{Fe}-$ ICR-7 and Al-ICR-7 are more solvothermally stable than corresponding ICR-6 MOFs. This behaviour can be rationalized by hydrophobicity of the phenyl groups pointing into the pore accessible volume, effectively shielding the coordination bonds of the linkers. ${ }^{29,30}$

We also investigated the stability of ICR MOFs towards activation from water, i.e., under conditions when wet MOFs are dried on air without the exchange of water for other solvent before drying. Some water-stable Zr-MOFs, such as PCN-222 or NU-10oo, loose porosity during the activation process from water. ${ }^{31}$ In contrast, phosphinate ICR MOFs, except for Al-ICR-6, display low variability in surface areas, indicating exceptional stability of the porous structure. The presented experimental results confirm that the ICR family of MOFs represents robust materials, well-suited for applications in aqueous environment. 
Table 2. Specific surface areas of as-prepared and treated ICR MOFs. ${ }^{a}$

\begin{tabular}{|c|c|c|c|c|c|c|c|c|}
\hline \multirow[b]{2}{*}{ Sample } & \multirow[b]{2}{*}{ As prepared } & \multicolumn{3}{|c|}{ Reflux } & \multicolumn{3}{|c|}{ RT } & \multirow{2}{*}{$\begin{array}{c}\text { Activated }^{\mathrm{b}} \\
\mathrm{H}_{2} \mathrm{O}\end{array}$} \\
\hline & & $\mathrm{H}_{2} \mathrm{O}$ & $\mathrm{EtOH}$ & Toluene & $\mathrm{H}_{2} \mathrm{O}$ & $\mathrm{EtOH}$ & Toluene & \\
\hline Fe-ICR-2 & 906 & 738 & 360 & 790 & 917 & 952 & 921 & 969 \\
\hline Fe-ICR-6 & 1134 & 172 & 1077 & 1081 & 1195 & 945 & 1174 & 1092 \\
\hline Fe-ICR-7 & 1125 & 896 & 1012 & 1061 & 1065 & 1122 & 1056 & 1077 \\
\hline Al-ICR-2 & 933 & 851 & 806 & 878 & 887 & 915 & 908 & 836 \\
\hline Al-ICR-6 & 1362 & 171 & 1444 & 1654 & 1087 & 1126 & 1212 & 747 \\
\hline Al-ICR-7 & 1030 & 701 & 1210 & 1076 & 1099 & 1164 & 1121 & 908 \\
\hline
\end{tabular}

${ }^{a}$ Specific surface areas are in $\mathrm{m}^{2} \mathrm{~g}^{-1}$. The t-plot method was used for Fe-ICR-2 and Al-ICR-2, otherwise BET specific surface areas are given. ${ }^{\mathrm{b}}$ Activation from water.

Adsorption of bisphenol A. The robustness, pore size variability, and hydrophobic nature of the pores prompted us to investigate the sorption properties of ICR MOFs towards hydrophobic pollutants. For these experiments, we selected Al-ICR MOFs as the porosity of Al-ICRs is greater than that of the $\mathrm{Fe}$ analogues and bisphenol $\mathrm{A}$ (BPA), a pollutant from the family of endocrine disruptors that represents significant threat in the food chain. The adsorption properties of Al-ICR MOFs were analyzed using high-performance liquid chromatography (HPLC). With the exception of Al-ICR-4 (PLD $2.9 \AA$ ), all other AlICR MOFs possess pores large enough to accommodate BPA molecules. The kinetic curves and adsorption isotherms of Al-ICR MOFs were compared with those of conventional activated charcoal (abbreviated as AC, Sigma-Aldrich), measured under identical conditions. Prior to all measurements the adsorbents were activated under vacuum at $80^{\circ} \mathrm{C}$ overnight.

The adsorption rate is an important factor for practical applications in environmental remediation. Figure 2 depicts the recorded kinetic curves. The kinetic parameters, including the correlation factors obtained by non-linear fitting to the pseudo-second order kinetic model, are summarized in Table 3 and Table S3, and the corresponding fits are presented in Figure S62. Interestingly, the sorption equilibrium for AC, Al-ICR-2, and Al-ICR-7 was nearly completed within $15 \mathrm{~min}$. In contrast, Al-ICR-6 behaved differently. The sorption kinetics indicates two consecutive processes, where a fast initial step is followed by a slow process, so that the equilibrium is not reached with the time frame of the sorption experiment (i.e., 36o min). This behaviour can be attributed to a slow rearrangement of BPA molecules inside the pores indicated by molecular modelling. These results show that BPA can be arranged in two positions in the pores of Al-ICR-6 (for details see below).

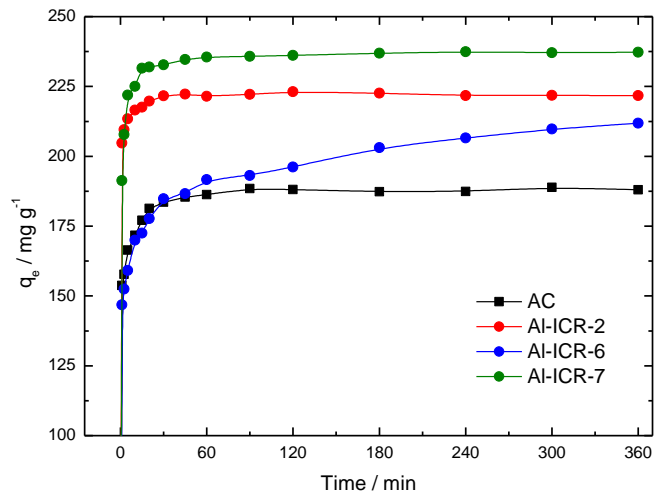

Figure 2. Kinetics of BPA adsorption by Al-ICR MOFs compared with that of AC. Conditions: initial BPA concentration $50 \mathrm{mg} \mathrm{L}^{-1}, 10 \mathrm{mg}$ of the adsorbent dispersed in $50 \mathrm{~mL} \mathrm{BPA}$ solution, $25 \pm 1{ }^{\circ} \mathrm{C}$. The experimental points are obtained from triplicate experiments (see Figure S62 for error bars).

Adsorption isotherms of BPA for Al-ICR MOFs and AC (Figure 3) were obtained using initial BPA concentrations from 10 to $120 \mathrm{mg} \mathrm{L}^{-1}$ after $24 \mathrm{~h}$ stirring at constant temperature $\left(25 \pm 1{ }^{\circ} \mathrm{C}\right)$. The experimental data were fitted using the Langmuir, Freundlich, and LangmuirFreundlich adsorption isotherm models (see the Supporting Information for details). Best fits were obtained using the Langmuir model providing parameters summarized in Table 3. The results indicate that the sorption capacity for $\mathrm{BPA}$ increases in the order Al-ICR-4 $<\mathrm{AC} \approx \mathrm{Al}-\mathrm{ICR}-2<\mathrm{Al}$ ICR-7 < Al-ICR-6. The highest adsorption capacity $\left(Q_{\mathrm{m}}\right)$ was found for Al-ICR-6 (326 mg g $)$, which is approximately by $50 \%$ greater value than the $Q_{\mathrm{m}}$ of AC (221 $\mathrm{mg} \mathrm{g}$ $\left.{ }^{1}\right)$. On the other hand, the adsorption capacity of Al-ICR-2 is comparable to that of $\mathrm{AC}$ and Al-ICR-4 adsorbs very little at the external surface due to the narrow pores (PLD of $2.9 \AA$ ). 


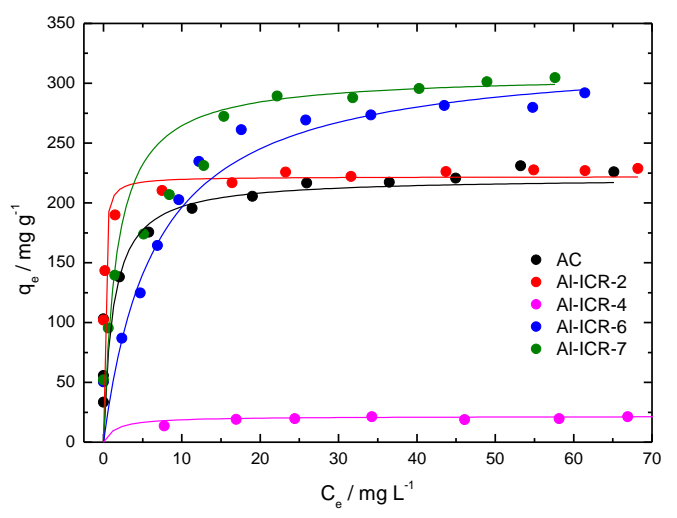

Figure 3. Adsorption isotherms of BPA expressed as the dependence of the adsorbed amount of BPA $\left(\mathrm{q}_{\mathrm{e}}\right)$ on the BPA equilibrium concentration $\left(C_{e}\right)$. The experimental points were obtained from triplicate experiments and the solid lines are the corresponding non-linear fits to the Langmuir adsorption model. For detail see the Supporting Information. Conditions: initial BPA concentration between 10 and 120 $\mathrm{mg} \mathrm{L}^{-1}$, $10 \mathrm{mg}$ of the adsorbent dispersed in $50 \mathrm{~mL}$ BPA solution, $25 \pm 1{ }^{\circ} \mathrm{C}$.

Table 3. Pseudo-second order kinetic constants and Langmuir isotherm constants obtained by non-linear fitting to the experimental data. ${ }^{a}$

\begin{tabular}{c|c|c|c|c}
\hline \multirow{2}{*}{ Sample } & \multicolumn{2}{|c|}{ Kinetic constants } & \multicolumn{2}{c}{ Langmuir constants } \\
\cline { 2 - 5 } & $\begin{array}{c}q_{\mathrm{m}} \\
\left(\mathrm{mg} \mathrm{g}^{-1}\right)\end{array}$ & $\begin{array}{c}k_{2} \\
\left(\mathrm{~g} \mathrm{mg}^{-1} \mathrm{~min}^{-1}\right)\end{array}$ & $\begin{array}{c}Q_{\mathrm{m}} \\
\left(\mathrm{mg} \mathrm{g}^{-1}\right)\end{array}$ & $\begin{array}{c}K_{\mathrm{L}} \\
\left(\mathrm{L} \mathrm{mg}^{-1}\right)\end{array}$ \\
\hline \hline AC & $183 \pm 2$ & $0.022 \pm 0.001$ & $221 \pm 4$ & $0.81 \pm 0.04$ \\
Al-ICR-2 & $220 \pm 7$ & $0.052 \pm 0.002$ & $222 \pm 3$ & $9.61 \pm 0.51$ \\
Al-ICR-4 & n. a. & n. a. & $22 \pm 1$ & $0.64 \pm 0.58$ \\
Al-ICR-6 & $194 \pm 4$ & $0.010 \pm 0.002$ & $326 \pm 8$ & $0.15 \pm 0.01$ \\
Al-ICR-7 & $234 \pm 1$ & $0.017 \pm 0.002$ & $307 \pm 5$ & $0.62 \pm 0.07$ \\
\hline
\end{tabular}

${ }^{\mathrm{a}}$ All data points were measured in triplicate experiments: $q_{\mathrm{m}}$ is the amount of BPA adsorbed at the equilibrium; $\mathrm{k}_{2}$ is the pseudo-second order kinetic rate constant; $Q_{\mathrm{m}}$ is the Langmuir maximum sorption capacity; $K_{\mathrm{L}}$ is the Langmuir constant.

Interestingly, the course of the adsorption isotherm of Al-ICR-2 is different from the isotherms of the other adsorbents. BPA was completely adsorbed from the dispersions with initial concentrations up to $30 \mathrm{mg} \mathrm{L}^{-1}$, and $\mathrm{Al}-$ ICR-2 became fully saturated at the initial BPA concentration of $50 \mathrm{mg} \mathrm{L}^{-1}$. On the other hand, Al-ICR-6, Al-ICR-7, and $\mathrm{AC}$ only partially removed BPA at low initial concentrations; however, due to the high pore volumes, the $Q_{m}$ values are greater than that of Al-ICR-2. This observation correlates well with the values of $K_{\mathrm{L}}$ constants of the Langmuir isotherms (Table 3), which are the measure of the adsorbent-adsorbate affinity. Thus, the high value for Al-ICR-2 $\left(K_{\mathrm{L}}=9.61\right)$ indicates high affinity of BPA towards Al-ICR-2. In contrast, the $K_{\mathrm{L}}$ values for Al-ICR-6 and AlICR-7 are by more than one order of magnitude lower (o.15 and 0.62, respectively), indicating that the affinity of BPA to ICR MOFs with larger pores is significantly lower when compared with that of Al-ICR-2.

The stability of MOFs in aqueous media is an important issue affecting their applicability. For this reason, we also characterized Al-ICR MOFs by PXRD and $\mathrm{N}_{2}$ adsorption isotherms after the sorption of $\mathrm{BPA}$ and regeneration done by washing with water and acetone (Figure S64 and Table $\mathrm{S}_{4}$ ). These characteristics are in line with the results presented above on the treatment Al-ICR MOFs with water at RT, confirming that Al-ICR MOFs are stable during the sorption experiments. In addition, the adsorption process is reversible, i.e., BPA can be washed out from the pores of Al-ICR MOFs with acetone (see the Supporting Information for details).

Summing up, Al-ICR MOFs are endowed with greater adsorption capacity than zeolites, graphene, imprinted polymers, montmorillonite, and other materials. ${ }^{5}$ In recent years, several MOFs were successfully tested as adsorbents of BPA. The $Q_{\mathrm{m}}$ values for typical carboxylatebased MOFs (such as Fe-MIL-10o, Cr-MIL-101) do not exceed $260 \mathrm{mg} \mathrm{g}^{-1}$. $^{2}$ Only Al-MIL-53 displays a similar maximum sorption capacity $\left(325 \mathrm{mg} \mathrm{g}^{-1}\right)$ to Al-ICR-6. ${ }^{33}$

Molecular modelling. We used molecular modelling in order to analyze the interactions of BPA inside the MOF pores. As described above, the pores are of hexagonal shape with the phenyl or methyl substituents bonded to $\mathrm{P}$ atoms aiming at the centre of the pore. These substituents along with the pore diameter can influence the interactions and arrangement of guest molecules as well as the sorption capacity.

The interaction energies between BPA and Al-ICR MOFs for relevant BPA amounts adsorbed in the pores are summarized in Table 4. At low concentrations of BPA in the framework (one BPA molecule per pore in the supercell, i.e., $\left.\mathrm{q}_{\mathrm{e}} \approx 10 \mathrm{mg} \mathrm{g}^{-1}\right)$, the interaction energies decrease in the order Al-ICR2 > Al-ICR-6 > Al-ICR-7. The snapshots of BPA arrangements are given in Figure 4a-c. The interaction energies in the Al-ICR-2 pores are nearly flat up to the loading of approximately $200 \mathrm{mg} \mathrm{g}^{-1}$. This behaviour is in good agreement with the observed high affinity of BPA towards Al-ICR-2, indicated by nearly quantitative adsorption of BPA at these concentrations and high $K_{\mathrm{L}}$ value.

Interestingly, there are two positions of BPA in the AlICR-6 pore (Figure S65) with an interaction energy difference of $2.9 \mathrm{kcal} \mathrm{mol}^{-1}$ (details in the Supporting Information). The existence of two binding sites and the relocation of BPA molecule between these two sites during simulation can be the reasons of the measured slow adsorption kinetics (Figure 2). At higher BPA concentrations (up to $\mathrm{q}_{\mathrm{e}} \approx 300 \mathrm{mg} \mathrm{g}^{-1}$ ), the interaction energy decreases due to BPA-BPA stacking interactions making the system quite disordered (Figure $4 \mathrm{~d}$ and Figure S66). The average interaction energies for Al-ICR-6 and Al-ICR-7 decrease with the increasing loading of BPA in agreement with the low $K_{L}$ values found for these materials (Table 3 ). 


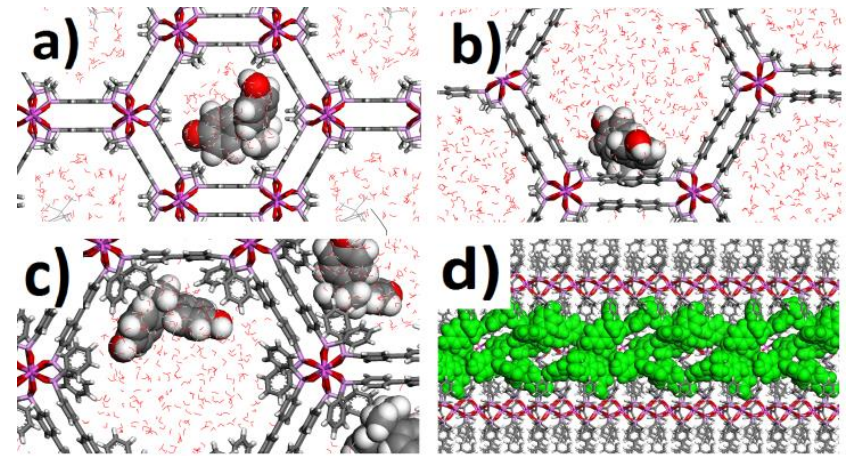

Figure 4. BPA molecule in the $1 \mathrm{D}$ pores of Al-ICR-2 (a), ICR6 (b), and ICR-7(c), view along the $c$ axes, $q_{e} \approx 10 \mathrm{mg} \mathrm{g}^{-1}$. Arrangement of BPA molecules in the $1 \mathrm{D}$ pore of Al-ICR- $6, \mathrm{q}_{\mathrm{e}}$ $\approx 30 \mathrm{mg} \mathrm{g}^{-1}$ view perpendicularly to the $\mathrm{c}$ axis $(\mathrm{d})$.

Table 4. Interaction energies between BPA and AlICR MOFs per one BPA molecule. ${ }^{a}$

\begin{tabular}{c|cccc}
\hline \multirow{2}{*}{ Sample } & \multicolumn{4}{|c}{$\mathrm{q}_{\mathrm{e}}\left(\mathrm{mg} \mathrm{g}^{-1}\right)$} \\
\cline { 2 - 5 } & 10 & 100 & 200 & 300 \\
\hline \hline Al-ICR-2 & $-22.2 \pm 0.4$ & $-21.3 \pm 0.3$ & $-21.2 \pm 0.3$ & n.a. \\
Al-ICR-6 & $-20.3 \pm 0.4^{\mathrm{b}}$ & $-17.7 \pm 0.2$ & $-17.6 \pm 0.2$ & $-16.4 \pm 0.2$ \\
Al-ICR-7 & $-18.2 \pm 0.4$ & $-16.3 \pm 0.3$ & $-16.3 \pm 0.2$ & $-15.3 \pm 0.5$ \\
\hline
\end{tabular}

${ }^{\mathrm{a}} \mathrm{q}_{\mathrm{e}}$ is the adsorbed amount of BPA per gram of Al-ICR $\mathrm{MOF}$, interaction energies are given in $\mathrm{kcal} \mathrm{mol}^{-1}$. ${ }^{\mathrm{b}}$ The interaction energy is the weighted average over two BPA positions shown in Figure. S65.

\section{CONCLUSIONS}

We synthesized new ICR-6 and ICR-7 MOFs isoreticular with Fe-ICR-2 and Fe-ICR-4 described earlier. ${ }^{20} \mathrm{We}$ also shown that $\mathrm{Al}^{3+}$ cations can be successfully used for the construction of ICR MOFs. ICR MOFs have high thermal and solvothermal stability. Due to the hydrophobic character of the ICR pore walls, ICR MOFs effectively adsorb BPA with greater sorption capacities than the majority of already investigated adsorbents.

Summing up, this work extends the area of phosphinic acid-based MOFs. The isoreticular design is applicable and the wide variety of water-stable MOFs can be prepared using various substituents at phosphorus atom. We envision that the number of phosphinic acid-based MOFs will steeply increase in coming years. ${ }^{34}$

\section{EXPERIMENTAL SECTION}

Preparation of Fe-ICR-2, Fe-ICR-4, Fe-ICR-7, and AlICR MOFs

A teflon lined autoclave (Berghof DAB-2) was charged with $0.08 \mathrm{mmol}$ linker and $0.04 \mathrm{mmol}$ of $\mathrm{FeCl}_{3} \cdot 6 \mathrm{H}_{2} \mathrm{O}(10.8$ $\mathrm{mg})$ or $\mathrm{AlCl}_{3} \cdot 6 \mathrm{H}_{2} \mathrm{O}(9.7 \mathrm{mg})$, and overlaid with $5 \mathrm{~mL}$ of absolute $\mathrm{EtOH}$. The sealed autoclave was heated in a preheated heating mantle (Berghof BTC-30oo) at $250{ }^{\circ} \mathrm{C}$ for $24 \mathrm{~h}$. The resulting white powder was centrifuged (11,0oo rpm, 5 min, Hettich, Rotina 380 R), washed five times with EtOH (third time it was left in $\mathrm{EtOH}$ for two hours), three times with water (second time it was left in water overnight), twice with acetone (third time it was left in acetone for one and half hours), and activated at 80 ${ }^{\circ} \mathrm{C}$ for five hours under vacuum.

\section{Preparation of Fe-ICR-5}

A teflon lined autoclave (Berghof DAB-2) was charged with $0.08 \mathrm{mmol}$ of $\mathrm{H}_{2} \mathrm{BBP}(\mathrm{Me})$ and $0.04 \mathrm{mmol} \mathrm{FeCl}_{3} \cdot 6 \mathrm{H}_{2} \mathrm{O}$ (10.8 mg), and overlaid with $5 \mathrm{~mL}$ of absolute EtOH. The sealed autoclave was heated in a preheated heating mantle (Berghof BTC-300o) at $250{ }^{\circ} \mathrm{C}$ for $24 \mathrm{~h}$. The resulting white powder was centrifuged (11,0oo rpm, 5 min, Hettich, Rotina $380 \mathrm{R}$ ), washed five times with acetone, and dried on air.

Preparation of Fe-ICR-6

A Wheaton vial was charged with $37.2 \mathrm{mg} \mathrm{H}_{2} \mathrm{BBP}(\mathrm{Me})$ (o.12 mmol) and overlaid with $25 \mathrm{~mL}$ of dimethylformamide (DMF). After $10 \mathrm{~min}$ sonication, $16.2 \mathrm{mg}$ of $\mathrm{FeCl}_{3} \cdot 6 \mathrm{H}_{2} \mathrm{O}(0.06 \mathrm{mmmol})$ in $5 \mathrm{ml}$ of DMF was added. The vial was heated in a preheated oven (Berghof BTC-30oo) at $120{ }^{\circ} \mathrm{C}$ for $72 \mathrm{~h}$. The resulting white powder was centrifuged (11,0oo rpm, $5 \mathrm{~min}$, Hettich, Rotina $380 \mathrm{R}$ ) and washed as described for Fe-ICR-2.

Stability of ICR MOFs

$20 \mathrm{mg}$ of MOF was suspended in $10 \mathrm{~mL}$ of $\mathrm{H}_{2} \mathrm{O}, \mathrm{EtOH}$, or toluene and the suspension was shaken for $24 \mathrm{~h}$ at RT or refluxed for $24 \mathrm{~h}$. After that, the solid material was collected by centrifugation, washed twice with water (only in the case of stability tests in water) or EtOH (in the case of stability tests in $\mathrm{EtOH}$ and toluene), and twice with acetone. The resulting powders were air-dried at RT.

\section{Adsorption of BPA}

The adsorption experiments were performed in sealed $100 \mathrm{~mL}$ reagent SIMAX glass bottles in a temperaturecontrolled room with constant temperature of $25 \pm 1{ }^{\circ} \mathrm{C}$ and BPA concentrations between 10 and $120 \mathrm{mg} \mathrm{L}^{-1}$. The bottles were charged with $10 \mathrm{mg}$ of Al-ICR MOF or activated charcoal $\left(A C, D_{A R C O}{ }^{\circledR}\right.$, 100 mesh particle size, powder, Sigma Aldrich) and $10 \mathrm{~mL}$ of water followed by $5 \mathrm{~min}$ sonication. Then, $40 \mathrm{~mL}$ of BPA solution was added. The mixture was stirred for $24 \mathrm{~h}$ at $25{ }^{\circ} \mathrm{C}$ and then $1 \mathrm{~mL}$ of sample was taken, filtered through a PTFE microfilter (o.2 $\mu \mathrm{m}$, Whatman), and the remaining concentration of BPA was analysed using HPLC-DAD.

\section{ASSOCIATED CONTENT}

Supporting Information. Detailed experimental procedures, FTIR, PXRD, Rietveld fits, TGA, and details for adsorption of BPA and molecular modelling (PDF). This material is available free of charge via the Internet at http://pubs.acs.org. 


\section{AUTHOR INFORMATION}

\section{Corresponding Author}

* E-mail: demel@iic.cas.cz

\section{ORCID}

Daniel Bůžek: oooo-ooo2-7387-9461

Jan Hynek: oooo-ooo3-1883-9464

Kamil Lang: oooo-0oo2-4151-8805

Jan Rohlíček: oooo-ooo1-6913-2667

Jan Demel: oooo-ooo1-7796-6338

\section{Notes}

The authors declare no competing financial interest.

\section{ACKNOWLEDGMENT}

This work was supported by the Czech Science Foundation (No. 20-04408S) and partial project DPK/2018/14 from the Technology Agency of the Czech Republic (GAMA COMNID TGo2010049). X-ray diffractometers were supported by the Operational Programme Research, Development and Education financed by the European Structural and Investment Funds and the Ministry of Education, Youth and Sports (No. SOLID21 CZ.02.1.01/o.o/o.o/16_019/oooo76o). The computational study was supported by the CESNET LM2015042 and the CERIT Scientific Cloud LM2015085, provided under the programme "Projects of Large Research, Development, and Innovations Infrastructures". The authors acknowledge the assistance provided by the Research Infrastructure NanoEnviCz, supported by the Ministry of Education, Youth and Sports of the Czech Republic under Project No. LM2015073.

\section{ABBREVIATIONS}

ICR Inorganic Chemistry Řež; MOF Metal-organic framework; SBU Secondary building unit; DMF $\mathrm{N}, \mathrm{N}$ dimethylformamide; PXRD Powder X-ray diffraction; PLD Pore limiting diameter; BET Brunauer-Emmett-Teller; NLDFT Non-local density functional theory; AC Activated charcoal; BPA Bisphenol A; HPLC High-performance liquid chromatography.

\section{REFERENCES}

(1) Nowak, K.; Jabłońska, E.; Ratajczak-Wrona, W. Immunomodulatory Effects of Synthetic Endocrine Disrupting Chemicals on the Development and Functions of Human Immune Cells. Environ. Int. 2019, 125, 350-364.

(2) Galloway, T. S.; Lee, B. P.; Burić, I.; Steele, A. M.; BPA Schools Study Consortium, Kocur, A. L.; Pandeth, A. G.; Harries L. W. in Plastics and the Environment, Hester R. E.; Harrison R. M. (Eds.), Royal Society of Chemistry, 2019, Chapter: Plastics Additives and Human Health: A Case Study of Bisphenol A (BPA), pp 131-155.

(3) Michałowicz, J. Bisphenol A - Source, toxicity and biotranformation. Environ. Toxicol. Pharmacol. 2014, 37, 738-758.

(4) Nam, S. H.; Seo, Y. M.; Kim, M. G. Bisphenol A Migration From Polycarbonate Babe Bottle with Repeated Use. Chemosphere 2010, 79, 949-952.

(5) Bhatnagar, A.; Anastopoulos, I. Adsorptive Removal of Bisphenol A (BPA) from Aqueous Solution: A Review. Chemosphere 2017, 168, 885-902.

(6) Wang, F.; Lu, X.; Peng, W.; Deng, Y.; Zhang, T.; Hu, Y.; Li, X. Y. Sorption Behavior of Bisphenol A and Triclosan by Graphene: Comparison with Activated Carbon. ACS Omega 2017, 2, $5378-5384$.
(7) Eddaoudi, M.; Kim, J.; Rosi, N.; Vodak, D.; Wachter, J.; O'Keeffe, M.; Yaghi, O. M. Systematic Design of Pore Size and Functionality in Isoreticular MOFs and their Application in Methane Storage. Science 2002, 295, 469-472.

(8) Guillerm, V.; Kim, D.; Eubank, J. F.; Luebke, R.; Liu, X.; Adil, K.; Lah, M. S.; Eddaoudi, M. A Supermolecular Building Approach for the Design and Construction of Metal-Organic Frameworks. Chem. Soc. Rev. 2014, 43, 6141-6172.

(9) Furukawa, H.; Cordova, K. E.; O'Keeffe, M.; Yaghi, O. M. The Chemistry and Applications of Metal-Organic Frameworks. Science 2013, 341, 1230444.

(10) Kim, D.; Liu, X.; Lah, M. S. Topology Analysis of MetalOrganic Frameworks Based on Metal-Organic Polyhedra as Secondary or Tertiary Building Units. Inorg. Chem. Front. 2015, 2, 336-36o.

(11) Koh, K; Wong-Foy, A. G.; Matzger, A. J. A Porous Coordination Copolymer with over $5000 \mathrm{~m}^{2} / \mathrm{g}$ BET Surface Area. J. Am. Chem. Soc. 2009, 131, 4184-4185.

(12) Farha, O. K.; Eryazici, I.; Jeong, N. C.; Hauser, B. G.; Wilmer, C. E.; Serjeant, A. A.; Snurr, R. Q.; Nguyen, S. T.; Yazaydin, A. Ö.; Hupp, J. T. Metal-Organic Framework Materials with Ultrahigh Surface Areas: Is the Sky the Limit? J. Am. Chem. Soc. 2012, 134, 15016-15021.

(13) Razavi, S. A. K.; Morsali, A. Linker Functionalized MetalOrganic Frameworks. Coord. Chem. Rev. 2019, 399, 213023.

(14) Cohen, S. M. Postsynthetic Methods for the Functionalization of Metal-Organic Frameworks. Chem. Rev. 2012, 112, 9701000.

(15) Pettinari, C.; Marchetti, F.; Mosca, N.; Tosi, G; Drozdov, A. Application of Metal - Organic Frameworks. Polym. Int. 2017, 66, 731-744.

(16) Drout, R. J.; Robison, L.; Chen, Z.; Islamoglu, T.; Farha, O. K. Zirconium Metal-Organic Frameworks for Organic Pollutant Adsoprtion. Trends in Chem. 2019, 1, 304-317.

(17) Bobbitt, N. S.; Mendonca, M. L.; Howarth, A. J.; Islamoglu, T.; Hupp, J. T.; Farha, O. K.; Snurr, R. Q. Metal-Organic Frameworks for the Removal of Toxic Industrial Chemicals and Chemical Warfare Agents. Chem. Soc. Rev. 2017, 46, 3357-3385.

(18) Feng, M.; Zhang, P.; Zhou, H.-C.; Sharma, V. K. WaterStable Metal-Organic Frameworks for Aqueous Removal of Heavy Metals and Radionuclides: A Review. Chemosphere, 2018, 209, 783-80o.

(19) Bůžek, D.; Demel, J.; Lang, K. Zirconium Metal-Organic Framework UiO-66: Stability in an Aqueous Environment and Its Relevance for Organophosphate Degradation. Inorg. Chem. 2018, 57, 14290-14297.

(2o) Hynek, J.; Brázda, P.; Rohlíček, J.; Londesborough, M. G. S.; Demel, J. Phosphinic Acid Based Linkers: Building Blocks in Metal-Organic Framework Chemistry. Angew. Chem. Int. Ed. 2018, 57, 5016 -5019.

(21) Serre, C.; Millange, F.; Thouvenot, C.; Noguès, M.; Marsolier, G.; Louër, D.; Férey, G.Very Large Breathing Effect in the First Nanoporous Chromium(III)-Based Solids: MIL-53 or $\mathrm{Cr}^{\mathrm{III}}(\mathrm{OH}) \cdot\left\{\mathrm{O}_{2} \mathrm{C}-\mathrm{C}_{6} \mathrm{H}_{4}-\mathrm{CO}_{2}\right\} \cdot\left\{\mathrm{HO}_{2} \mathrm{C}-\mathrm{C}_{6} \mathrm{H}_{4}-\mathrm{CO}_{2} \mathrm{H}\right\}_{\mathrm{x}} \cdot \mathrm{H}_{2} \mathrm{O}_{\mathrm{y}}$. J. Am. Chem. Soc. 2002, 124, 13519-13526.

(22) Program DICVOL 06: https://doi.org/10.1107/So021889804014876

(23) Program FOX: https://doi.org/10.339o/cryst7100322

(24) Materials Studio Modeling Environment, Release 4.3 Documentation. Accelrys Software Inc., San Diego, CA, 2003.

(25) Palatinus, L.; Chapuis, G. Superflip - a Computer Program for the Solution of Crystal Structures by Charge Flipping in Arbitrary Dimensions. J. Appl. Cryst. 2007, 40, 786-79o.

(26) Sarkisov, L.; Harrison, A. Computational Structure Characterisation Tools in Application to Ordered and Disordered Porous Materials. Mol. Simul. 2011, 37, 1248-1257. 
(27) Poreblazer v3.0.2 (2017): http://www.homepages.ed.ac.uk/lsarkiso/Research.html

(28) Lawrence, M. C.; Schneider, C.; Katz, M. J. Determining the Structural Stability of UiO-67 with Respect to Time: a SolidState NMR Investigation, Chem. Commun. 2o16, 52, 4971-4974.

(29) Bosch, M.; Zhang, M.; Zhou, H.-C. Increasing the Stability of Metal-Organic Frameworks. Adv. Chem. 2014, 182327.

(30) Burtch, N. C.; Jasuja, H.; Walton, K. S. Water Stability and Adsorption in Metal-Organic Frameworks, Chem. Rev. 2014, 114, 10575-10612.

(31) Mondloch, J. E.; Katz, M. J.; Planas, N.; Semrouni, D.; Gagliardi, L.; Hupp, J. T.; Farha, O. K. Are $\mathrm{Zr}_{6}$-based MOFs Water Stable? Linker Hydrolysis vs. Capillary-Force-Driven Channel Collapse, Chem. Commun. 2014, 50, 8944-8946.
(32) Qin, F.-X; Jia, S.-Y.; Liu, Y.; Li, H.-Y.; Wu, S.-H. Adsorption Removal of Bisphenol A from Aqueous Solution Using MetalOrganic Frameworks. Desalin. Water Treat. 2015, 54, 93-102.

(33) Zhou, M.; Wu, Y.-N.; Qiao, J.; Zhang, J.; McDonald, A.; Li, G.; Li, F. The Removal of Bisphenol A from Aqueous Solution by MIL-53(Al) and Mesostructured MIL-53(Al) J. Colloid Interf. Sci. 2013, 405, 157-163.

(34) Nakatsuka, S.; Watanabe, Y.; Kamakura, Y.; Horike, S.; Tanaka, D.; Hatakeyama, T. Solvent-Vapor-Induced Reversible Single-Crystal-to-Single-Crystal Transformation of a Triphosphaazatriangulene-Based Metal-Organic Framework. Angew. Chem. Int. Ed. DOI: 10.1002/anie.201912195 


\section{Insert Table of Contents artwork here}

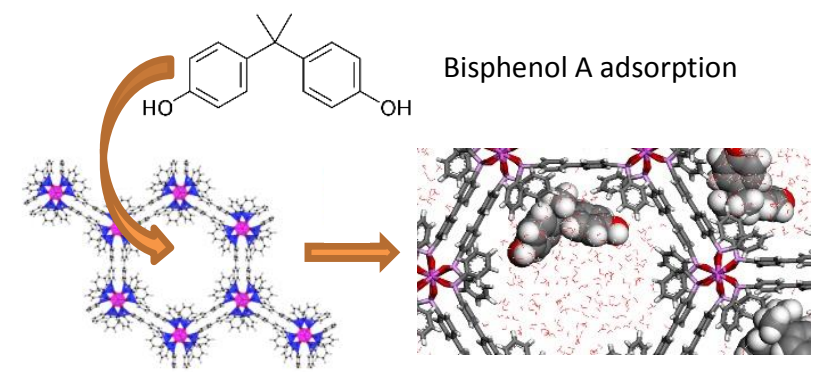

Isoreticular design of phosphinic

acid-based ICR MOFs 